\title{
Robust Estimation-Based Control Strategies for Induction Motors
}

\author{
Florin Stîngă, ${ }^{1}$ Marius Marian, ${ }^{2}$ and Dan Selişteanu $\mathbb{D}^{1}$ \\ ${ }^{1}$ Department of Automatic Control and Electronics, University of Craiova, A.I. Cuza No. 13, Craiova, RO 200585, Romania \\ ${ }^{2}$ Department of Computers and Information Technology, University of Craiova, A.I. Cuza No. 13, Craiova, RO 200585, Romania
}

Correspondence should be addressed to Dan Selişteanu; dansel@automation.ucv.ro

Received 18 January 2020; Accepted 26 June 2020; Published 29 July 2020

Academic Editor: Chongyang Liu

Copyright (C 2020 Florin Stîngă et al. This is an open access article distributed under the Creative Commons Attribution License, which permits unrestricted use, distribution, and reproduction in any medium, provided the original work is properly cited.

This work proposes a realistic solution to the control problem of sensorless induction motors. Due to some important aspects related to their construction and reliability, the induction motors are extensively used in many modern industrial applications. Considering that the system is facing the lack of hardware sensors, the proposed complex control strategies are based on the estimation of unavailable system variables and parameters. In order to control the rotor speed, two robust control strategies are proposed: a modified super-twisting adaptive technique and a model predictive technique. The tests performed under several practical assumptions show that the closed loop behaviour of the system is adequate, and the output variable follows the imposed time varying reference, despite the considered uncertainties and disturbances acting on the process.

\section{Introduction}

Nowadays, induction motors are facing an interesting challenge from the perspective of modelling and sensorless control. This is mainly caused by some particular, inherited operating conditions. In the last decades, due to the environmental rules imposed by the international institutions, the induction motors have been proposed to be a reliable solution for the usual drive systems.

Regarding the control design of these systems, beside the classical scalar control and vector control strategies [1-3], in the last years modern approaches have been proposed, such as input-output linearization and nonlinear/sliding mode/ nonlinear predictive control strategies [4-6].

Two specific problems are found in practice: first, the models are uncertain $[7,8]$ and, second, reliable physical sensors for the real-time measurements of process states [9] are unavailable. The developed control strategies use the "software sensors" paradigm, as an achievable combination between software estimators/observers and hardware sensors [10-13].

The present work approaches a linked observer-estimator used to estimate the unmeasurable state and those parameters that are uncertain or unknown. The proposed reduced-order state observer is designed by using an appropriate linear transformation and provides the reconstruction of rotor fluxes. In what concern the estimation of unknown process parameters (e.g., the stator resistance) and of the load torque, acting as an external disturbance on the rotor, a parameter estimator and a disturbance observer were developed. The parameter estimator is derived from a typical one used in biotechnology applications $[14,15]$. The disturbances observer provides an estimation result which can be used within a robust observer-based control method [16, 17].

Using the estimates provided by the proposed observers, two control strategies were proposed: a modified supertwisting algorithm (STA) and a robust model predictive control (RMPC), designed such that the output (i.e., rotor speed) follows a chosen time-varying reference.

The main objective of the super-twisting algorithm proposed by Levant in his work [18] is to reduce the chattering effect occurring in classical sliding mode control. Moreover, the algorithm must ensure the convergence and also resolve, in finite time, the tracking problem. In the recent studies, some practical and theoretical modified approaches of the original algorithm were proposed: adaptive gains super-twisting algorithm (AGSTA) used to provide some compensation of the smooth, bounded uncertainties and disturbances of the linear time invariant systems [19], multivariable super-twisting sliding mode 
structure, used to build an observer designed to detect faults for a satellite system [20], second-order super-twisting sliding mode controller (SOSM, STSMC) designed to deal with linear growing perturbations, in terms of robustness and finite time convergence [21-23], robust super-twisting algorithm for nonlinear systems [24], and adaptive supertwisting sliding mode control [25].

A super-twisting algorithm is typically used to impose zero values to the sliding variable and its time derivate in a finite time, to remove the chattering effect and to preserve the robustness by improving the disturbances rejection performance.

Our proposed approach uses an adaptive gain in the definition of the sliding surface in order to cope with timevarying disturbances acting on the system.

The second considered control algorithm is an optimal one, named model predictive control. This algorithm prevailed as an efficient method in widespread applications due to its optimal characteristics and some inherent features concerning the stability and robustness [26-28]. To form the predictions, the proposed strategy utilizes a discrete linearized model of the system [29]. The objective function casts the disturbances variable, such that the robustness of the controller is improved. Also, some input constraints are considered, such that the physical restrictions are fulfilled.

The main contributions of the paper consist in the design of a linked estimator-observer for unmeasurable/unknown variables of the process, and in the development of two novel modified robust control strategies that use the "software" information provided by the designed observers.

To emphasize the estimation, tracking, and robustness performances of the proposed algorithms, several realistic tests were performed, and some metrics defined in accordance with the tracking error were computed.

The combination of estimation and control algorithms leads to complex structures necessary for the general control objective. Because it is necessary to use combined information "software" from estimators and "hardware" from sensors, the general control structure must provide reliable solutions to problems related to convergence and to robustness (it is considered that the system is one subject to external disturbances). Also, the complexity of considered control strategies is an intrinsic one: the super-twisting algorithm requires a correct definition of the slip surface and the choices of the tuning parameters, due to the nonlinearities introduced by the control law, and moreover the predictive algorithm requires solving a minimization problem with constraints.

\section{Process Description and Control Objective}

2.1. Process Description. The fifth-order dynamical model of an induction motor [2] is considered:

$$
\begin{aligned}
& \frac{\mathrm{d}}{\mathrm{d} t} i_{d s}(t)=-a_{1} i_{d s}(t)-a_{2} i_{d s}(t) R_{s}(t)+\omega_{s} i_{q s}(t)+a_{3} a_{4} \phi_{d r}(t)+p a_{4} \omega_{r}(t) \phi_{q r}(t)+a_{5} u_{d s}(t), \\
& \frac{\mathrm{d}}{\mathrm{d} t} i_{q s}(t)=-\omega_{s} i_{d s}(t)-a_{1} i_{q s}(t)-a_{2} i_{q s}(t) R_{s}(t)+a_{3} a_{4} \phi_{q r}(t)-p a_{4} \omega_{r}(t) \phi_{d r}(t)+a_{5} u_{q s}(t), \\
& \frac{\mathrm{d}}{\mathrm{d} t} \phi_{d r}(t)=a_{3} L_{m} i_{d s}(t)-a_{3} \phi_{d r}(t)+\left(\omega_{s}-p \omega_{r}(t)\right) \phi_{q r}(t), \\
& \frac{\mathrm{d}}{\mathrm{d} t} \phi_{q r}(t)=a_{3} L_{m} i_{q s}(t)-a_{3} \phi_{q r}(t)-\left(\omega_{s}-p \omega_{r}(t)\right) \phi_{d r}(t), \\
& \frac{\mathrm{d}}{\mathrm{d} t} \omega_{r}(t)=\frac{p L_{m}}{J L_{r}}\left(\phi_{d r}(t) i_{q s}(t)-\phi_{q r}(t) i_{d s}(t)\right)-\frac{B}{J} \omega_{r}(t)-\frac{1}{J} T_{L}(t),
\end{aligned}
$$

where $i_{d s}$ and $i_{q s}, \phi_{d r}$ and $\phi_{q r}$, and $u_{d s}$ and $u_{q s}$ are the stator currents, rotor fluxes, and stator voltages; $p$ represents the number of poles pairs; $\omega_{r}$ is the rotor speed; $\omega_{s}$ is the synchronous speed; $T_{L}$ is the load torque; $B$ is the viscous coefficient; and $J$ is the rotor inertia:

$$
\begin{aligned}
& a_{1}=\frac{L_{m}^{2} R_{r}}{L_{r}\left(L_{s} L_{r}-L_{m}^{2}\right)}, \\
& a_{2}=\frac{L_{r}}{L_{s} L_{r}-L_{m}^{2}}, \\
& a_{3}=\frac{R_{r}}{L_{r}},
\end{aligned}
$$

$a_{4}=\frac{L_{m}}{L_{s} L_{r}-L_{m}^{2}}, \quad a_{5}=a_{2}, \quad \omega_{s}=2 \pi \cdot f$,

where $R_{r}, L_{r}, R_{s}$, and $L_{s}$ are the rotor/stator resistances and inductances; $L_{m}$ is the magnetizing inductance; and $f$ is the frequency of the voltage source.

Remark 1. The parameter $R_{s}$ is time varying, and the factors that affect this variation are the slip frequency and winding temperature during operation, with consequence during the process control stage [7].

For systems (1a)-(1e), considering relations (1a)-(1d), the next state space representation can be highlighted: 


$$
\dot{x}(t)=A x(t)+B_{1} u(t)+K_{1} R_{s}(t) x(t)+K_{2} \delta(x, t),
$$

where $x=\left[\begin{array}{llll}i_{d s} & i_{q s} & \phi_{d r} & \phi_{q r}\end{array}\right]^{T}$ is the vector of states, $u=$ $\left[\begin{array}{ll}u_{d s} & u_{q s}\end{array}\right]^{T}$ is the vector of control inputs, $\delta(x, t)=\left[\begin{array}{ll}\phi_{q r} \omega_{r} & \phi_{d r} \omega_{r}\end{array}\right]^{T}$ is a smooth nonlinear function, and

$$
\begin{aligned}
A & =\left[\begin{array}{cccc}
-a_{1} & \omega_{s} & a_{3} a_{4} & 0 \\
-\omega_{s} & -a_{1} & 0 & a_{3} a_{4} \\
a_{3} L_{m} & 0 & -a_{3} & \omega_{s} \\
0 & a_{3} L_{m} & -\omega_{s} & -a_{3}
\end{array}\right], \\
B_{1} & =\left[\begin{array}{cccc}
a_{5} & 0 & 0 & 0 \\
0 & a_{5} & 0 & 0
\end{array}\right]^{T}, \\
\mathrm{~K}_{1} & =\left[\begin{array}{cccc}
-a_{2} & 0 & 0 & 0 \\
0 & -a_{2} & 0 & 0 \\
0 & 0 & 0 & 0 \\
0 & 0 & 0 & 0
\end{array}\right], \\
\mathrm{K}_{2} & =\left[\begin{array}{cccc}
p a_{4} & 0 & -p & 0 \\
0 & -p a_{4} & 0 & p
\end{array}\right]^{T},
\end{aligned}
$$

are constant known matrices.

2.2. Control Objective. For the process described above, the objective is to control the rotor speed $\left(\omega_{r}\right)$ such that it follows certain reference values despite the external disturbances exerted on the rotor (the load torque $T_{L}$ ) and the time variation of a process parameter (the stator resistance $R_{s}$ ).

Remark 2. Some practical assumptions are considered:

(i) The rotor fluxes and the controlled variable are unmeasurable

(ii) The induction machine operates in a synchronous reference frame $(d-q)$

Thus, the controlled variable is the rotor speed; that is, $y=\omega_{r}$. The stator voltages represent the control input, so that $u=\left[\begin{array}{ll}u_{d s} & u_{q s}\end{array}\right]^{T}$, where $u_{d s} \in\left[0, V_{\max }\right], u_{q s} \approx 0$.

Therefore, we can formulate the following control problem: the considered output will asymptotically track some desired trajectories despite any external disturbances and uncertainties related to some time-varying process parameters and state variables (unknown or unmeasurable). To resolve this problem, we introduce state and disturbances observers as well as a parameter estimator, considering practical and technical hypotheses. Then, by means of these observers, we derive two control strategies. In order to capture the behaviour of the closed loop systems from practical operating conditions point of view, it was considered that a measurement noise acts on the input variables.

\section{Design of Specific Observers}

We assume that we have hardware sensors for the stator currents along the $d-q$ axes; therefore, the following partitions are defined: $x_{1}=\left[\begin{array}{ll}i_{d s} & i_{q s}\end{array}\right]^{T}$ is the vector of measured variables, and $x_{2}=\left[\begin{array}{ll}\varphi_{d s} & \varphi_{q s}\end{array}\right]^{T}$ is the vector of variables that have to be estimated.

3.1. A Linked Asymptotic State Observer: Observer-Based Estimator. For the process described by the dynamical model (1a)-(1e), the design of an asymptotic state observer is performed under the next hypotheses [30]:

$H 1$. Some matrices are known, namely, $K, A$, and $B_{1}$ $H 2$. The stator resistance $\left(R_{s}\right)$ is unknown

H3. A subset $n_{1}=2$ of states are measured in real-time

Under $H 3$ hypothesis, the model (3) can be described by

$$
\begin{aligned}
\dot{x}_{1}(t)= & A_{11} x_{1}(t)+A_{12} x_{2}(t)+B_{11} u(t)+K_{11} R_{s}(t) x_{1}(t) \\
& +K_{21} \delta(x, t), \\
\dot{x}_{2}(t)= & A_{21} x_{1}(t)+A_{22} x_{2}(t)+B_{12} u(t)+K_{12} R_{s}(t) x_{2}(t) \\
& +K_{22} \delta(x, t),
\end{aligned}
$$

where $\quad A_{11}=\left[\begin{array}{cc}-a_{1} & \omega_{s} \\ -\omega_{s} & -a_{1}\end{array}\right], \quad A_{12}=\left[\begin{array}{cc}a_{3} a_{4} & 0 \\ 0 & a_{3} a_{4}\end{array}\right]$,

$A_{21}=\left[\begin{array}{cc}a_{3} L_{m} & 0 \\ 0 & a_{3} L_{m}\end{array}\right], A_{22}=\left[\begin{array}{cc}-a_{3} & \omega_{s} \\ -\omega_{s} & -a_{3}\end{array}\right], B_{11}=\left[\begin{array}{cc}a_{5} & 0 \\ 0 & a_{5}\end{array}\right]$, $B_{12}=0_{2 \times 2}, \quad K_{11}=\left[\begin{array}{cc}-a_{2} & 0 \\ 0 & -a_{2}\end{array}\right], \quad K_{21}=\left[\begin{array}{cc}p a_{4} & 0 \\ 0 & p a_{4}\end{array}\right], \quad$ and $K_{12}=0_{2 \times 2}, K_{22}=\left[\begin{array}{cc}-p & 0 \\ 0 & p\end{array}\right]$.

We will use an appropriate linear transformation:

$$
z(t)=x_{2}(t)-K_{22} K_{21}^{-1} x_{1}(t),
$$

where the auxiliary variables vector is $z \in \mathfrak{R}^{2}$. Consequently, the dynamics of $z$ is

$$
\begin{aligned}
\dot{z}(t)= & \dot{x}_{2}(t)-K_{22} K_{21}^{-1} \dot{x}_{1}(t) \\
= & A_{21} x_{1}(t)+A_{22} x_{2}(t)+B_{12} u(t)+K_{12} R_{s}(t) x_{2}(t) \\
& +K_{22} \delta(x, t)-K_{22} K_{21}^{-1}\left(A_{11} x_{1}(t)+A_{12} x_{2}(t)+B_{11} u(t)\right. \\
& \left.+K_{11} R_{s}(t) x_{1}(t)+K_{21} \delta(x, t)\right) \\
= & A_{1} x_{1}(t)+A_{2} x_{2}(t)+A_{3} R_{s}(t) x_{1}(t) \\
& +A_{4} R_{s}(t) x_{2}(t)+B_{1} u(t),
\end{aligned}
$$

where $A_{1}=\left(A_{21}-K_{22} K_{21}^{-1} A_{11}\right), A_{2}=\left(A_{22}-K_{22} K_{21}^{-1} A_{12}\right)$, $A_{3}=-K_{22} K_{21}^{-1} K_{11}, \quad A_{4}=K_{12}=0_{2 \times 2}$, and $B_{1}=\left(B_{12}-K_{22}\right.$ $\left.K_{21}^{-1} B_{11}\right)$. 
From (5) and (6), the following asymptotic observer is defined:

$$
\begin{aligned}
\dot{\hat{z}}(t) & =A_{2} \widehat{z}(t)+A_{5} x_{1}(t)+A_{3} \widehat{R}_{s}(t) x_{1}(t)+B_{1} u(t), \\
x_{2}(t) & =\widehat{z}(t)+K_{22} K_{21}^{-1} x_{1}(t),
\end{aligned}
$$

where $\left(\hat{)}\right.$ denote estimated values and $A_{5}=A_{1}+A_{2} K_{22} K_{21}^{-1}$.

The performance of the observer ( 8 ) is obtained by using a Luenberger approach $[31,32]$ :

$$
\begin{aligned}
\dot{\hat{z}}(t)= & A_{2} \widehat{z}(t)+A_{5} x_{1}(t)+A_{3} \widehat{R}_{s}(t) x_{1}(t)+B_{1} u(t) \\
& +L(z(t)-\widehat{z}(t)), \\
x_{2}(t)= & \widehat{z}(t)+K_{22} K_{21}^{-1} x_{1}(t),
\end{aligned}
$$

where the gain matrix $L \in \mathfrak{R}^{2 \times 2}$ provides the tool to set the eigenvalues in the chosen positions such that the observer's convergence is assured.

Recall the hypothesis $H 2$; then, a solution to estimate the unknown parameter $R_{s}$ is provided by an appropriate observer-based estimator (OBE).

Let us define the positive definite Lyapunov candidate function:

$$
V(t)=e_{1}^{T}(t) \Gamma e_{1}(t)+e_{2}^{2}(t),
$$

where $\quad e_{1}(t)=z(t)-\widehat{z}(t), \quad e_{2}(t)=R_{s}(t)-\widehat{R}_{s}(t), \quad$ and $\Gamma>0 \in \mathfrak{R}^{2 \times 2}, \Gamma=\operatorname{diag}\left(\gamma_{1}, \gamma_{2}\right)$.

Thus, $\dot{V}(t)$ is expressed by

$$
\dot{V}(t)=e_{1}(t) \Gamma \dot{e}_{1}^{T}(t)+e_{1}^{T}(t) \Gamma \dot{e}_{1}(t)+2 e_{2}(t) \dot{e}_{2}(t),
$$

where

$$
\begin{aligned}
\dot{e}_{1}(t)= & \dot{z}(t)-\dot{\vec{z}}(t)=A_{1} x_{1}(t)+A_{2}\left(z(t)+K_{22} K_{21}^{-1} x_{1}(t)\right) \\
& +A_{3} R_{s}(t) x_{1}(t)+B_{1} u(t)-A_{2} \widehat{z}(t) \\
& -A_{5} x_{1}(t)-A_{3} \widehat{R}_{s}(t) x_{1}(t)-B_{1} u(t)-L(z(t) \\
& -\widehat{z}(t))=\left(A_{2}-L\right) e_{1}(t)+A_{3} x_{1}(t) e_{2}(t), \\
\dot{e}_{2}(t)= & \dot{R}_{s}(t)-\dot{\widehat{R}}_{s}(t) .
\end{aligned}
$$

Therefore,

$$
\begin{aligned}
\dot{V}= & e_{1} \Gamma\left[\left(A_{2}-L\right) e_{1}+A_{3} x_{1} e_{2}\right]^{T}+e_{1}^{T} \Gamma\left[\left(A_{2}-L\right) e_{1}\right. \\
& \left.+A_{3} x_{1} e_{2}\right]+2 e_{2}\left(\dot{R}_{s}-\dot{\widehat{R}}_{s}\right) \\
= & e_{1} \Gamma\left[\left(A_{2}-L\right) e_{1}\right]^{T}+e_{1} \Gamma\left[A_{3} x_{1} e_{2}\right]^{T}+e_{1}^{T} \Gamma\left[\left(A_{2}-L\right) e_{1}\right] \\
& +e_{1}^{T} \Gamma\left[A_{3} x_{1} e_{2}\right]+2 e_{2}\left(\dot{R}_{s}-\dot{\hat{R}}_{s}\right) .
\end{aligned}
$$

The OBE is defined, without loss of generality, under the next hypotheses:

H4. $\dot{V}(t)<0, \forall t>0$

H5. $\left(A_{2}-L\right)$ is a Hurwitz stable matrix

H6. $\dot{R}_{s}(t)$ is considered bounded, such that $\left\|\dot{R}_{s}(t)\right\| \leq \varepsilon$, for all $t \geq 0$, and $\varepsilon$ is a positive real constant
H7. $\lim _{t \rightarrow \infty} \dot{R}_{s}(t)=0$

Then, from $(H 4-H 7)$, the equation,

$$
\begin{aligned}
& e_{1}(t) \Gamma\left(A_{3} x_{1}(t) e_{2}(t)\right)^{T}+e_{1}^{T}(t) \Gamma\left(A_{3} x_{1}(t) e_{2}(t)\right) \\
& \quad+2 e_{2}(t)\left(-\dot{\widehat{R}}_{s}(t)\right)=0,
\end{aligned}
$$

must be fulfilled.

Thus, for the initial nonlinear system, the following linked observer-estimator is proposed:

$$
\begin{aligned}
\dot{\widehat{z}}(t)= & A_{2} \widehat{z}(t)+A_{5} x_{1}(t)+A_{3} \widehat{R}_{s}(t) x_{1}(t)+B_{1} u(t) \\
& +L(z(t)-\widehat{z}(t)), \\
x_{2}(t)= & \widehat{z}(t)+K_{22} K_{21}^{-1} x_{1}(t), \\
\dot{\widehat{R}}_{s}(t)= & \left(A_{3} x_{1}(t)\right)^{T} \Gamma(z(t)-\widehat{z}(t)) .
\end{aligned}
$$

Moreover, based on $(H 4-H 7)$, the estimation errors $e_{1}$ and $e_{2}$ vanish to zero as $t \longrightarrow \infty$.

3.2. Disturbances Observer. To estimate the possible unknown external disturbances (the load torque), the following observer is proposed for the initial nonlinear model [33]:

$$
\dot{v}(t)=-L_{1} b_{2}(v(t)+v(t))
$$

$$
-L_{1}\left(\frac{p L_{m}}{J L_{r}}\left(\widehat{\phi}_{d r}(t) i_{q s}(t)-\widehat{\phi}_{q r}(t) i_{d s}(t)\right)-\frac{B}{J} \omega_{r}(t)\right),
$$

$\widehat{T}_{L}(t)=v(t)+v(t)$,

where $v \in \Re$ and $v \in \Re$, are auxiliary variables, $L_{1} \in \Re$ is a gain parameter used to achieve the convergence of the observer, $b_{2}=-(1 / J), \widehat{\varphi}_{d r}$, and $\widehat{\varphi}_{q r}$ are provided by the observer (15).

Let $e_{3}(t)=\widehat{T}_{L}(t)-T_{L}(t)$. Then, the error dynamics has the next expression:

$$
\begin{aligned}
\dot{e}_{3}(t) & =\dot{\widehat{T}}_{L}(t)-\dot{T}_{L}(t) \\
& =-L_{1} b_{2}(v(t)+v(t))-L_{1}\left(\dot{x}_{3}(t)-b_{2} T_{L}(t)\right)+\dot{v}(t)-\dot{T}_{L}(t) \\
& =-L_{1} b_{2} \widehat{T}_{L}(t)-L_{1} \dot{x}_{3}(t)+L_{1} b_{2} T_{L}(t)+\dot{v}(t)-\dot{T}_{L}(t) \\
& =-L_{1} b_{2}\left(\widehat{T}_{L}(t)-T_{L}(t)\right)-L_{1} \dot{x}_{3}(t)+\dot{v}(t)-\dot{T}_{L}(t) \\
& =-L_{1} b_{2} e_{3}(t)-L_{1} \dot{x}_{3}(t)+\dot{v}(t)-\dot{T}_{L}(t) .
\end{aligned}
$$

If the following hypotheses hold,

H8. The term $-L_{1} b_{2}<0$

H9. The auxiliary variable $v(t)=L_{1} x_{3}(t)$

H10. $\dot{T}_{L}(t)$ is bounded, such that $\left\|\dot{T}_{L}(t)\right\| \leq \varepsilon$, $\forall t \geq 0, \varepsilon>0$

H11. $\lim _{t \rightarrow \infty} \dot{T}_{L}(t)=0$

then, $\lim _{t \rightarrow \infty} e_{3}(t)=0$.

Based on observer (15), the dynamics of the unmeasurable output variable is defined by 


$$
\frac{\mathrm{d}}{\mathrm{d} t} \widehat{\omega}_{r}(t)=\frac{p L_{m}}{J L_{r}}\left(\widehat{\phi}_{d r}(t) i_{q s}(t)-\widehat{\phi}_{q r}(t) i_{d s}(t)\right)-\frac{B}{J} \widehat{\omega}_{r}(t)-\frac{1}{J} \widehat{T}_{L}(t) .
$$

\section{Design of Robust Estimation- Based Controllers}

Under the previous assumptions (see Section 2.2), we will develop two robust control strategies: a super-twisting algorithm and a model predictive algorithm.

4.1. Modified Super-Twisting Control Algorithm. We define an appropriate sliding surface [24]:

$$
s(t)=\dot{e}_{y}(t)+l_{1} e_{y}(t)+l_{2} \int_{0}^{t} e_{y}(\tau) \mathrm{d} \tau
$$

where $e_{y}=\widehat{\omega}_{r}(t)-\omega_{r}^{*}(t), \omega_{r}^{*}(t)$ denotes the reference trajectory, $l_{1}$ and $l_{2}$ are positive constants, and $\widehat{\omega}_{r}$ is given by relation (18).

The control law is a defined as

$$
u_{d}(t)=u_{1}(t)+u_{2}(t)
$$

where $u_{1}(t)$ is equivalent control of the system, considering $T_{L}(t)=0$, and $u_{2}(t)$ is designed by using a super-twisted control algorithm.

The time derivative of (20) yields that

$$
\begin{aligned}
\dot{s}(t)= & \ddot{e}_{y}(t)+l_{1} \dot{e}_{y}(t)+l_{2} e_{y}(t) \\
& =\left(\ddot{\widehat{\omega}}_{r}(t)-\ddot{\omega}_{r}^{*}(t)\right)+l_{1}\left(\dot{\widehat{\omega}}_{r}(t)-\dot{\omega}_{r}^{*}(t)\right)+l_{2}\left(\widehat{\omega}_{r}(t)-\omega_{r}^{*}(t)\right) .
\end{aligned}
$$

Using relations (1a)-(1e), (15), and (18) and considering $u_{q s} \approx 0$, the above equation leads to the following expansion:

$$
\begin{aligned}
\dot{s}(t)= & \frac{p L_{m}}{J L_{r}}\left(\frac{\mathrm{d}}{\mathrm{d} t}\left(\widehat{\phi}_{d r}\right) \cdot i_{q s}+\widehat{\phi}_{d r} \cdot \frac{\mathrm{d}}{\mathrm{d} t}\left(i_{q s}\right)\right. \\
& \left.-\frac{\mathrm{d}}{\mathrm{d} t}\left(\widehat{\phi}_{q r}\right) \cdot i_{d s}-\widehat{\phi}_{q r} \cdot \frac{\mathrm{d}}{\mathrm{d} t}\left(i_{d s}\right)\right)-\frac{B}{J} \cdot \frac{\mathrm{d}}{\mathrm{d} t}\left(\widehat{\omega}_{r}\right)- \\
& -\frac{\mathrm{d}^{2}}{\mathrm{~d} t^{2}} \omega_{r}^{*}+l_{1}\left(\frac{p L_{m}}{J L_{r}}\left(\widehat{\phi}_{d r} \cdot i_{q s}-\widehat{\phi}_{q r} \cdot i_{d s}\right)\right. \\
& \left.-\frac{B}{J} \cdot\left(\widehat{\omega}_{r}\right)-\frac{\mathrm{d}}{\mathrm{d} t} \omega_{r}^{*}\right)+l_{2}\left(\widehat{\omega}_{r}-\omega_{r}^{*}\right)=0 .
\end{aligned}
$$

Thus,

$$
u_{1}(t)=u_{d s}(t)=\frac{1}{g(x, t)}(-f(x, t)-r(t))
$$

where

$$
\begin{aligned}
f(x, t)= & \frac{p L_{m}}{J L_{r}}\left(\frac{\mathrm{d}}{\mathrm{d} t}\left(\widehat{\phi}_{d r}\right) \cdot i_{q s}+\widehat{\phi}_{d r} \cdot \frac{\mathrm{d}}{\mathrm{d} t}\left(i_{q s}\right)\right. \\
& -\frac{\mathrm{d}}{\mathrm{d} t}\left(\widehat{\phi}_{q r}\right) \cdot i_{d s}-\widehat{\phi}_{q r} \cdot\left(-a_{1} \cdot i_{d s}-a_{2} \cdot i_{d s} \cdot \widehat{R}_{s}+\right. \\
& \left.\left.+\omega_{s} \cdot i_{q s}+a_{3} \cdot a_{4} \cdot \widehat{\phi}_{d r}+p \cdot a_{4} \cdot \widehat{\omega}_{r}\right)\right)-\frac{B}{J} \cdot \frac{\mathrm{d}}{\mathrm{d} t}\left(\widehat{\omega}_{r}\right)+l_{2} \cdot \widehat{\omega}_{r}, \\
g(x, t)= & -\frac{p L_{m}}{J L_{r}} \cdot a_{5} \cdot \widehat{\phi}_{q r}, \\
r(t)= & -\frac{\mathrm{d}^{2}}{\mathrm{~d} t^{2}} \omega_{r}^{*}-l_{1}\left(\frac{\mathrm{d}}{\mathrm{d} t} \omega_{r}^{*}\right)-l_{2}\left(\omega_{r}^{*}\right) .
\end{aligned}
$$

Remark 3. The existence of the abovementioned control component $\left(u_{1}\right)$ is guaranteed if $\widehat{\phi}_{q r} \neq 0$.

In the literature, usually, the super-twisting controller has the following form $[20,22,24]$ :

$$
\begin{aligned}
u_{2}(t) & =-l_{3}|s(t)|^{1 / 2} \operatorname{sgn}(s(t))-l_{4} s(t)+a(t), \\
\dot{a}(t) & =-l_{5} \operatorname{sgn}(s(t))-l_{6} s(t),
\end{aligned}
$$

where $l_{3}, l_{4}, l_{5}$, and $l_{6}$ are positive constants.

Remark 4. The convergence of system (25) along with sufficient conditions was proven (see, e.g., [20, 24]).

However, in accordance with our physical restrictions imposed for the control input, we propose the following modified form of the relation:

$$
u_{d}(t)= \begin{cases}\left(u_{1}(t)+u_{2}(t)\right), & \text { if }\left(0<u_{1}(t) \leq V_{\max }\right), \\ \left(u_{2}(t)\right), & \text { if }\left(u_{1}(t)>V_{\max }\right), \\ V_{\max }, & \text { if }\left(u_{d}(t)>V_{\max }\right), \\ 0, & \text { if }\left(u_{d}(t) \leq 0\right) .\end{cases}
$$

Moreover, to improve the robustness of considered controller, we will suppose that the gain $l_{2}$ (see relation (19)) is time varying. In fact, we consider that $l_{2}\left(t, \widehat{T}_{L}\right)=c \cdot \widehat{T}_{L}(t)$, where $c \in(0,(1 / J)]$ such that a proper compensation of the external disturbances occurs.

Therefore, the vector of control inputs can be expressed as $u=\left[\begin{array}{ll}u_{d} & u_{q s}\end{array}\right]^{T}=\left[\begin{array}{ll}u_{d} & 0\end{array}\right]^{T}$.

4.2. Design of the Robust Model-Predictive Controller. The discrete model which will be used is a linear approximation of system (3), obtained by using Taylor expansion. The linear model is

$$
\dot{\tilde{x}}(t)=A_{1}^{l} \widetilde{x}(t)+A_{2}^{l} \widetilde{R}_{s}(t)+B_{1}^{u} \widetilde{u}(t)+B_{1}^{w} \widetilde{T}_{L}(t),
$$

where $\tilde{x}(t)=\left[i_{d s}(t)-\bar{i}_{d s} i_{q s}(t)-\bar{i}_{q s} \phi_{d r}(t)-\bar{\phi}_{d r} \phi_{q r}(t)-\right.$ $\left.\bar{\phi}_{q r} \omega_{r}(t)-\bar{\omega}_{r}\right]^{T}, \quad \widetilde{R}_{s}=\widehat{R}_{s}(t)-\bar{R}_{s}, \quad \widetilde{T}_{L}=\widehat{T}_{L}(t)-\bar{T}_{L}$, with $\widehat{R}_{s}(t)$ and $\widehat{T}_{L}$ provided by the observers (15) and (16), 
$\tilde{u}(t)=\left[\begin{array}{l}u_{d s}(t)-\bar{u}_{d s} \\ u_{q s}(t)-\bar{u}_{q s}\end{array}\right]=\left[\begin{array}{c}u_{d}(t)-\bar{u}_{d} \\ u_{q s}(t)-\bar{u}_{q s}\end{array}\right]$, where

$u_{d}(t)=u_{d s}(t)$ and $\bar{u}_{d}=\bar{u}_{d s}$,

$$
A_{1}^{l}=\left[\begin{array}{ccccc}
-a_{2} \bar{R}_{s}-a_{1} & \omega_{s} & a_{3} a_{4} & p a_{4} \bar{\omega}_{r} & p a_{4} \bar{\phi}_{q r} \\
-\omega_{s} & -a_{2} \bar{R}_{s}-a_{1} & -p a_{4} \bar{\omega}_{r} & a_{3} a_{4} & -p a_{4} \bar{\phi}_{d r} \\
a_{3} L_{m} & 0 & -a_{3} & \left(\omega_{s}-p \bar{\omega}_{r}\right) & -p \bar{\phi}_{q r} \\
0 & a_{3} L_{m} & -\left(\omega_{s}-p \bar{\omega}_{r}\right) & -a_{3} & p \bar{\phi}_{d r} \\
-\frac{p L_{m}}{J L_{r}} \bar{\phi}_{q r} & \frac{p L_{m}}{J L_{r}} \bar{\phi}_{d r} & \frac{p L_{m}}{J L_{r}} \bar{i}_{q s} & -\frac{p L_{m}}{J L_{r}} \bar{i}_{d s} & -\frac{B}{J}
\end{array}\right],
$$

$A_{2}^{l}=\left[\begin{array}{lllll}-a_{2} \bar{i}_{d s} & -a_{2} \bar{i}_{q s} & 0 & 0 & 0\end{array}\right]^{T}, B_{1}^{u}$ and $B_{1}^{w}$ were defined in the previous section, and $\left(^{-}\right)$denotes the equilibrium states. The equilibrium states can be determined as

$$
\begin{aligned}
& P\left(\bar{\omega}_{r}\right)\left[\begin{array}{llll}
\bar{i}_{d s} & \bar{i}_{q s} & \bar{\phi}_{d r} & \bar{\phi}_{q r}
\end{array}\right]^{T}=B_{1}\left[\begin{array}{ll}
\bar{u}_{d} & 0
\end{array}\right]^{T}, \\
& \bar{R}_{S}=\frac{p L_{m}}{L_{r}}\left(\bar{i}_{q s} \bar{\phi}_{d r}-\bar{i}_{d s} \bar{\phi}_{q r}\right)-B \bar{\omega}_{r},
\end{aligned}
$$

where

$$
\left[\begin{array}{cccc}
-a_{2} \bar{R}_{s}-a_{1} & \omega_{s} & a_{3} a_{4} & p a_{4} \bar{\omega}_{r} \\
-\omega_{s} & -a_{2} \bar{R}_{s}-a_{1} & -p a_{4} \bar{\omega}_{r} & a_{3} a_{4} \\
a_{3} L_{m} & 0 & -a_{3} & \left(\omega_{s}-p \bar{\omega}_{r}\right) \\
0 & a_{3} L_{m} & -\left(\omega_{s}-p \bar{\omega}_{r}\right) & -a_{3}
\end{array}\right] \text { and } B_{1}
$$

was defined in Section 2.1.

So, by imposing $\bar{\omega}_{r}, \bar{R}_{s}$, and $\bar{u}_{d}$, we determine the equilibrium points as

$$
\left[\begin{array}{llll}
\bar{i}_{d s} & \bar{i}_{q s} & \bar{\phi}_{d r} & \bar{\phi}_{d r}
\end{array}\right]^{T}=P^{-1}\left(\bar{\omega}_{r}\right) B_{1}\left[\begin{array}{ll}
\bar{u}_{d} & 0
\end{array}\right]^{T} \text {. }
$$

We define the discrete-time model:

$$
\begin{aligned}
\eta(k+1) & =A_{1}^{v} \eta(k)+A_{2}^{v} \widetilde{R}_{s}(k)+B_{u} \Delta u(k)+B_{w} \widetilde{T}_{L}(k), \\
y(k) & =C v(k),
\end{aligned}
$$

where $\eta(k)=\left[\begin{array}{ll}\Delta x(k)^{T} & y(k)\end{array}\right]^{T}, \Delta x(k)=\tilde{x}(k)-\tilde{x}(k-1)$, $\Delta u(k)=\tilde{u}(k)-\tilde{u}(k-1), \quad A_{1}^{v}=\left[\begin{array}{cc}A_{1}^{d} & 0_{n \times p} \\ C A_{1}^{d} & I_{p \times p}\end{array}\right], \quad A_{2}^{v}=$ $\left[\begin{array}{c}A_{2}^{d} \\ C A_{2}^{d}\end{array}\right], B_{u}=\left[\begin{array}{c}B_{1}^{d} \\ C B_{1}^{d}\end{array}\right], B_{w}=\left[\begin{array}{c}B_{2}^{d} \\ C B_{2}^{d}\end{array}\right], C=\left[\begin{array}{ll}0_{p_{1} \times n} & I_{p_{1} \times p_{1}}\end{array}\right]$, with $p_{1}$ outputs and $n$ state variables.

The matrices $A_{i}^{d}=I+A_{i}^{l} T_{e}, i=1,2, \quad$ and $B_{i}^{d}=B_{i}^{l} T_{e}, i=1,2$, where $t=k T_{e}$ (for sake of simplicity $t=k)\left(T_{e}\right.$ is the sample time) are obtained by Euler approximation.

Let us consider the constrained minimization problem:

$$
\begin{array}{ll}
\min _{\Delta U} & \frac{1}{2} \Delta U\left(T \Xi_{u}^{T} \bar{Q}_{1} \Xi_{u}+\bar{Q}_{3}\right) \Delta U+\left(\left(\Xi_{u}^{T} \bar{Q}_{1} F\right) \eta+\left(\Xi_{u}^{T} \bar{Q}_{2} \Xi_{R_{s}}\right) \Delta R+\left(\Xi_{u}^{T} \bar{Q}_{2} \Xi_{T_{L}}\right) \Delta T_{L}-\left(\Xi_{u}^{T} \bar{Q}_{1}\right) Y_{r}\right)^{T} \Delta U \\
\text { subject to } & \left(\left[\begin{array}{c}
-\Omega_{1} \\
\Omega_{1}
\end{array}\right] \Delta U \leq\left[\begin{array}{c}
-\Delta U_{\min }+\Omega_{2} u(k-1) \\
\Delta U_{\max }-\Omega_{2} u(k-1)
\end{array}\right]\right),
\end{array}
$$

where 


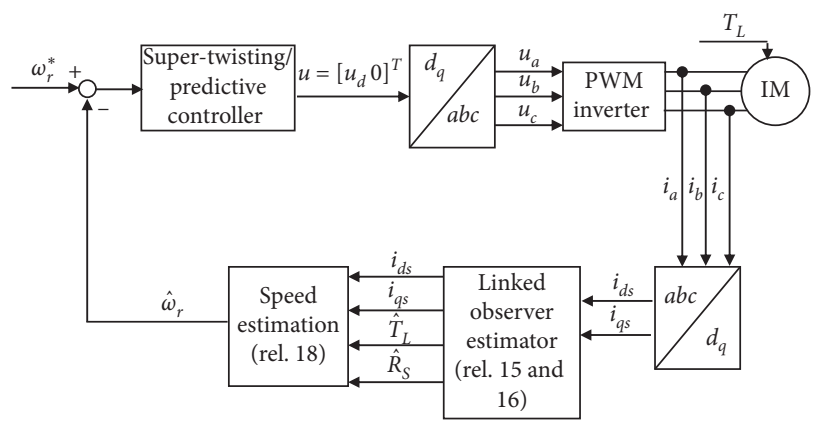

FIgURE 1: The estimation and control scheme proposed for the induction machine.

$$
\begin{aligned}
\Delta U & =\left[\Delta u(k), \ldots, \Delta u\left(k+N_{c}-1\right)\right]^{T}, \\
\Delta R & =\left[\widetilde{R}_{s}(k), \ldots, \widetilde{R}_{s}\left(k+N_{c}-1\right)\right]^{T}, \\
\Delta T_{L} & =\left[\widetilde{T}_{L}(k), \ldots, \widetilde{T}_{L}(k)\left(k+N_{c}-1\right)\right]^{T} .
\end{aligned}
$$

where $N_{c}$ is the control horizon, $\Omega_{1}$ is a $\left(m \cdot N_{c}\right) \times(m$. $N_{c}$ ) lower triangular matrix, where $m$ is number of inputs, $\Omega_{2}$ is a matrix with $N_{c}$ identity matrix $I_{m \times m}, \Delta U_{\min }$ and $\Delta U_{\max }$ are vectors with $\left(m \cdot N_{c}\right)$ elements of $u_{\min }$ and $u_{\max }$, respectively, and $\Xi_{u}$ is a lower triangular matrix with the nonzero elements defined by

$$
\xi_{i, j}=C\left(A_{g}^{v}\right)^{i-j} B_{u}, \quad g=1,2 ; i=\overline{1, N_{p}} ; j=\overline{1, N_{c}},
$$

where $N_{p}$ is the prediction horizon; $Y_{r}$ is a vector with $p$. $N_{p}$ elements of reference values; $\bar{Q}_{1}, \bar{Q}_{2}$, and $\bar{Q}_{3}$, are positive definite weight matrices; and $F$ is a matrix with the elements:

$$
f_{i}=\left(C\left(A_{1}^{v}\right)^{i}\right)^{T}, \quad i=1, \ldots, N_{p} .
$$

Problem (32) was solved by using Hildreth's quadratic programming algorithm, so that the adjustment of component of the Lagrange multiplier vector, $\mu$, is given by [34]

$$
\mu_{i}^{k+1}=\max \left(0, \beta_{i}^{k+1}\right)
$$

with

$$
\beta_{i}^{k+1}=-\frac{1}{\varepsilon_{i i}}\left[o_{i}+\sum_{j=1}^{i-1} \varepsilon_{i j} \mu_{j}^{k+1}+\sum_{j=i+1}^{n} \varepsilon_{i j} \mu_{j}^{k}\right],
$$

where $\varepsilon_{i j}$ is the $i j$ th element in the matrix $\mathrm{E}=M H^{-1} M^{T}$ and $o_{i}$ is the $i$ th element in the vector $O=N+M H^{-1} F$.

Then, the solution is

$$
\Delta U=-H^{-1}\left(F+M^{T} \mu\right) .
$$

Moreover, the receding horizon strategy [29] allows that only the first term of the sequence (38) is considered at step $k$.

The sufficient conditions (proper terminal cost, adequate prediction horizon, and so on) to ensure the convergence of the closed loop system were defined in [35].

The general scheme of proposed estimation and control is depicted in Figure 1.

\section{Results and Discussion}

Estimator (15) was examined in open loop using models (1a)-(1e). The values of process parameters are as follows [13]: $R_{r}=44 \mathrm{~m} \Omega, L_{r}=22 \mathrm{mH}, L_{s}=22 \mathrm{mH}, L_{m}=21.5 \mathrm{mH}$, $p=3, f=50 \mathrm{~Hz}, J=5 \mathrm{~kg} \cdot \mathrm{m}^{2}, B=0.1 \mathrm{Nm} / \mathrm{rad} / \mathrm{sec}$, and $u_{\text {max }}=380 \mathrm{~V}$, and the considered initial conditions are $i_{d s}(0)=i_{q s}(0)=0 \mathrm{~A}, \quad \phi_{d r}(0)=\phi_{q r}(0)=0 \mathrm{~Wb}, \quad R_{s}(0)=$ $68 \mathrm{~m} \Omega, u_{d s}=200 \mathrm{~V}$, and $u_{q s}=0 \mathrm{~V}$.

It is worth noting that for the abovementioned values, matrix $A_{2}$ of the asymptotic observer (8), which determines the convergence propriety, has imaginary eigenvalues: $\lambda_{1,2}= \pm j 314.1593$. Such eigenvalues lead to an oscillatory behaviour and eventually to instability. Instead, if the extended form of the observer was used (see relation (15)), with the gain matrix determined by pole placement method $L=\left[\begin{array}{cc}500 & 314.1593 \\ -314.1593 & 500\end{array}\right]$, then the observer matrix $A_{2}-$ $L$ has the chosen eigenvalues as $\lambda_{1,2}=-500$.

The tuning matrix, used for the estimation of $R_{s}$, has the form $\Gamma=\left[\begin{array}{cc}0.05 & 0 \\ 0 & 5\end{array}\right], \quad z(0)=\left[\widehat{\phi}_{d r}(0) \widehat{\phi}_{q r}(0)\right]^{T}=[0.40 .5]^{T}$ $\mathrm{Wb}$, and $\widehat{R}_{s}(0)=40 \mathrm{~m} \Omega$. For the disturbances observer, the tracking parameter is $L_{1}=-1000$, and the initial condition is $\widehat{T}_{L}(0)=60 \mathrm{Nm}$

The estimations of the unmeasured state variables, of the unknown variables $R_{s}$ and $T_{L}$, obtained by using linked observer-estimator (15), are presented in Figures 2 and 3, respectively. Figure 4 presents the evolution of unmeasured output variable provided by relation (18). A nonzero initial condition is considered, as $\widehat{\omega}_{r}(0)=2 \mathrm{rad} / \mathrm{sec}$.

The presented graphics show an asymptotic convergence of the proposed observer despite the variation of the stator resistance and the considered external disturbance of the process, represented by the load torque exerted on the rotor.

The behaviour in closed loop with the proposed control strategies (super-twisting and predictive) is presented in Figures 5 and 6 . These figures show the evolution of the output estimation $\left(\widehat{\omega}_{r}\right)$, compared with the considered timevarying reference profile.

The control strategies were carried out using the following tuning parameters and weighting matrices (obtained by trial and error method) and the control input constraints: 


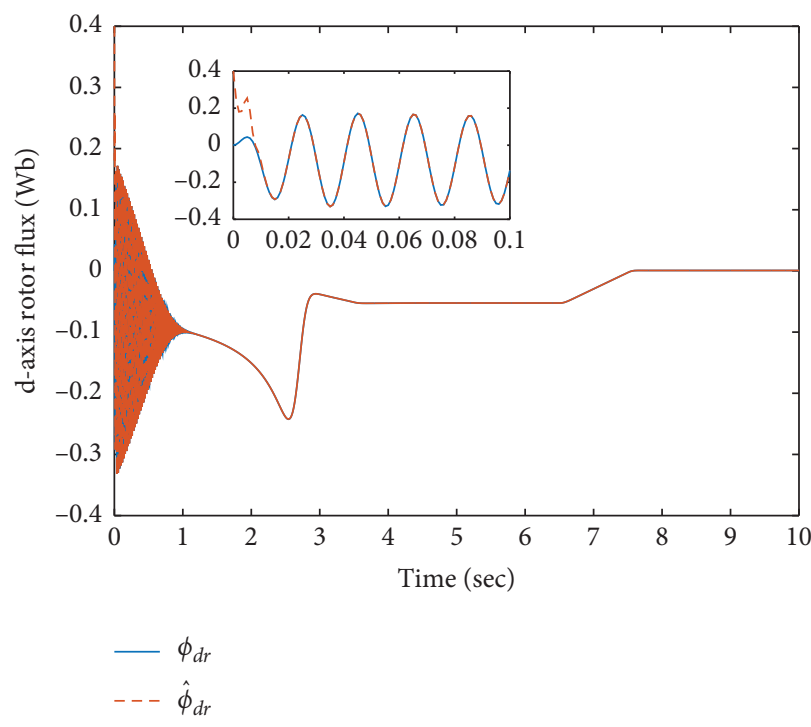

(a)

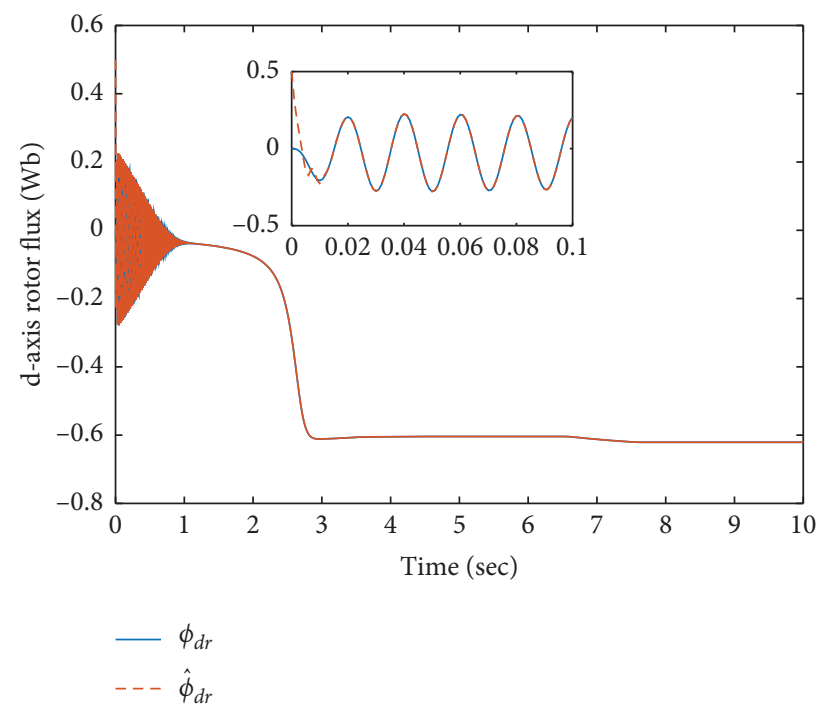

(b)

FIgURE 2: Evolution of $\phi_{d r}$ and $\phi_{q r}$ versus their estimates provided by (15).

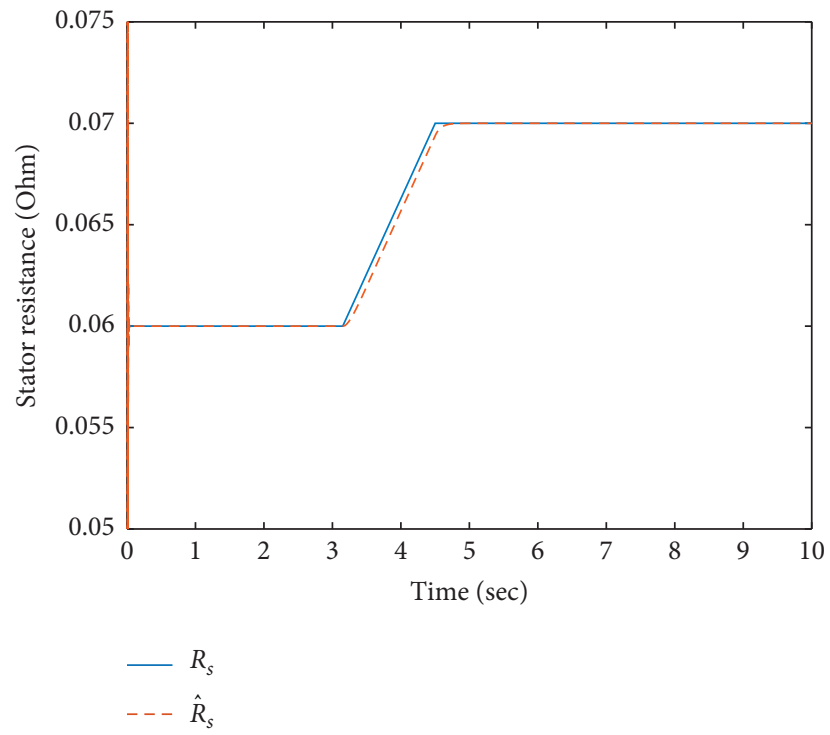

(a)

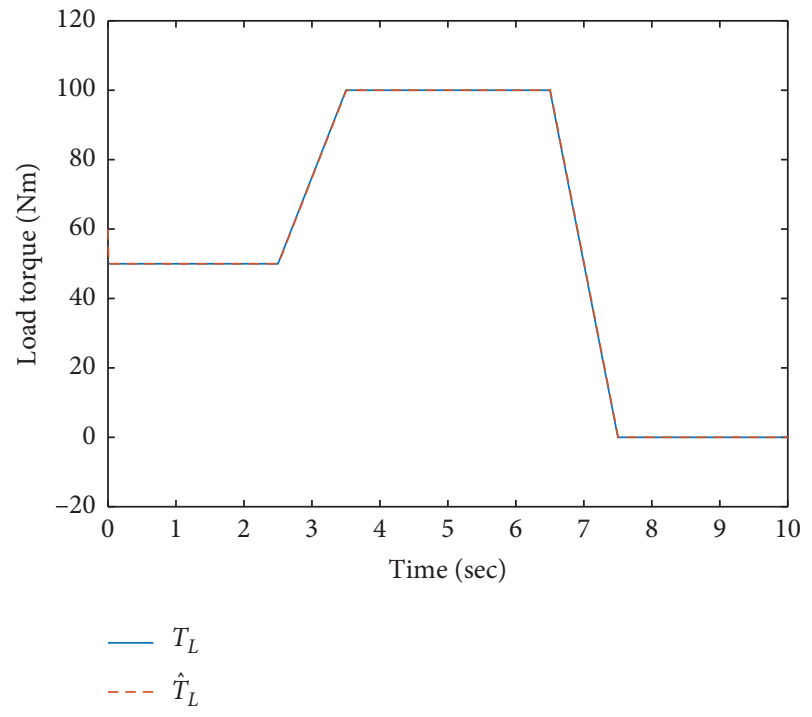

(b)

Figure 3: Evolution of $R_{s}$ and $T_{L}$, respectively, and of their estimates given by observer (15).

(i) Super-twisting controller: $l_{1}=1000, c=0.2, l_{3}=5$, $l_{4}=0.001, l_{5}=5$, and $l_{6}=0.001$

(ii) Predictive controller: $N_{c}=10, N_{p}=15, \bar{Q}_{1}=7 \cdot 10^{6}$. $I_{N_{p} \times N_{p}}, \bar{Q}_{2}=1 \cdot 10^{3} \cdot I_{N_{p} \times N_{p}, T}, \bar{Q}_{3}=8 \cdot 10^{3} \cdot \quad I_{2 N_{c} \times 2 N_{c}}$, $T_{e}=0.001 \mathrm{sec}, u_{\min }=\left[\begin{array}{ll}0 & 0\end{array}\right]^{T}$, and $u_{\max }=\left[\begin{array}{lll}380 & 0\end{array}\right]^{T^{2}}$

The equilibrium points used in linearization process were determined by relation (30), considering the following values: $\left\{\bar{\omega}_{r}, \bar{R}_{s}, \bar{u}_{d s}, \bar{u}_{q s}\right\}=\{90 \mathrm{rad} / \mathrm{sec}, 0.068 \Omega, 200 \mathrm{~V}, 0 \mathrm{~V}\}$.

The first considered scenario is the "ideal" case, when the measured variables (the stator currents) are not perturbed.
Figures 7 and 8 present the evolution of control inputs applied to the process. It is pointed out that the inputs are bounded according to our physical working conditions. Figure 9 depicts the components of the control input on $\mathrm{d}$-axis determined by relation (26).

From the presented graphics, it can be noticed that the control laws have the ability to maintain the output close to its reference. The control aim was attained even if for the design we used less a priori information about the process and despite the considered variation of the process parameter (the stator resistance, see Figure 10) and the time- 


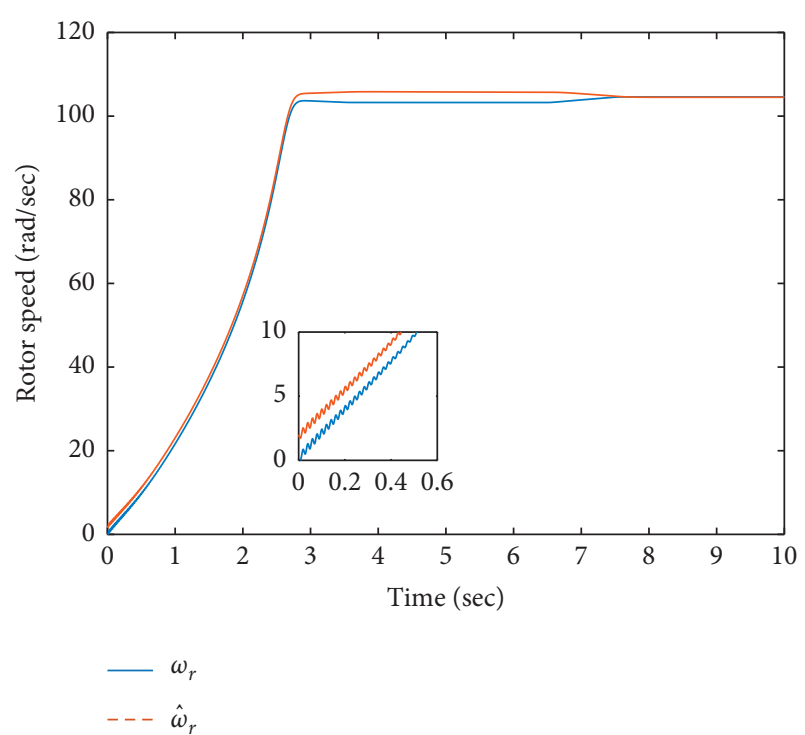

FIGURE 4: The time profile of $\widehat{\omega}_{r}$.

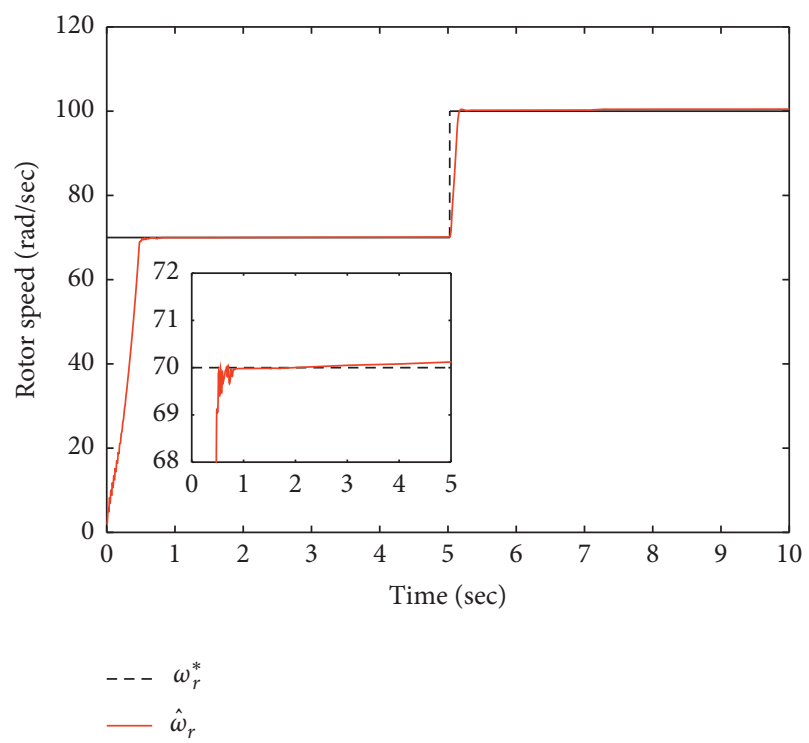

FIGURE 5: Evolution of output by using the proposed super-twisting control algorithm.

varying profile of the considered disturbances (the load torque), presented in Figure 11.

Moreover, the second scenario aims to verify the robustness of control algorithms, from another perspective: the presence of noise in the acquisition of the measurable variables (the stator currents). It is considered that the measurements are corrupted with white noise (variation of $10 \%$ from their nominal values). The tuning parameters and weighting matrices were described above.

The evolutions of the output variable and control effort are presented in Figures 12-15, respectively. From these graphics it can be observed that even in the case of the perturbed measurable variables, the control algorithms react very well. As it was already observed from the previous

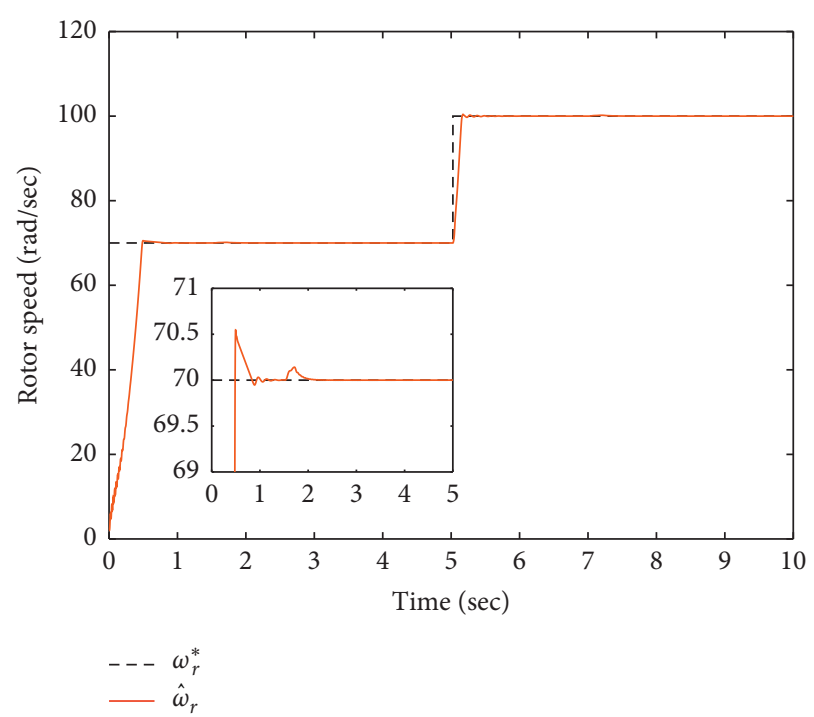

FIgURE 6: Evolution of output by using the proposed robust predictive control algorithm.

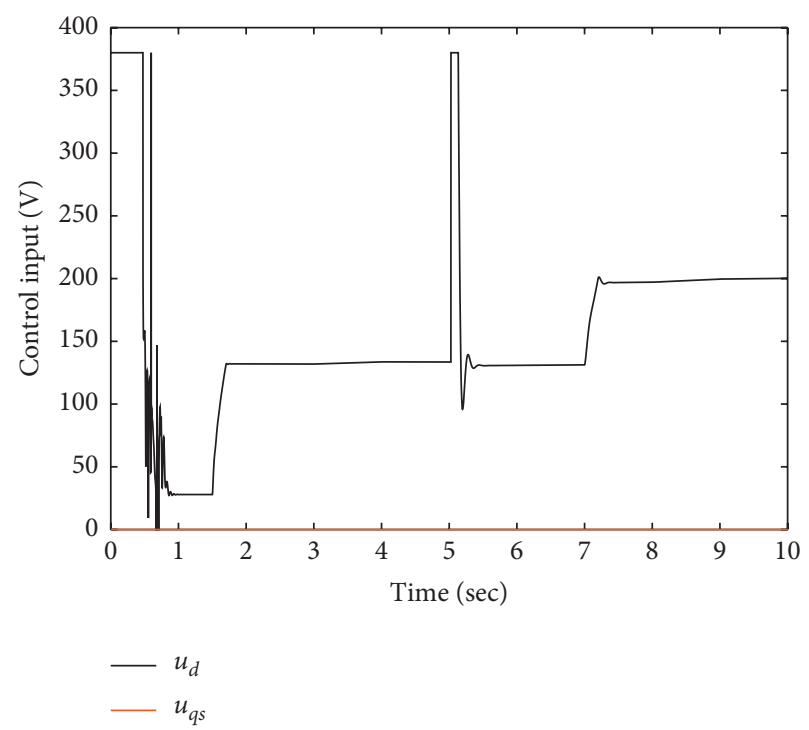

FIgURE 7: Evolution of control inputs.

scenario, better results are obtained in the predictive case, regarding robustness and tracking problems.

Figure 16 presents the evolution of measured variables disturbed by noise. The graphic is obtained for the predictive control strategy.

The tests were accomplished in the MATLAB environment [36].

For a better comparison, the controlled system performance was also analysed by using some metrics, defined in accordance with the tracking error as $e_{y}=\widehat{\omega}_{r}(t)-\omega_{r}^{*}(t)$. The following performance indices were calculated $[37,38]$ :

(i) Integral time absolute error, ITAE $=\int_{0}^{T_{f}} t\left|e_{y}(t)\right| \mathrm{d} t$ 


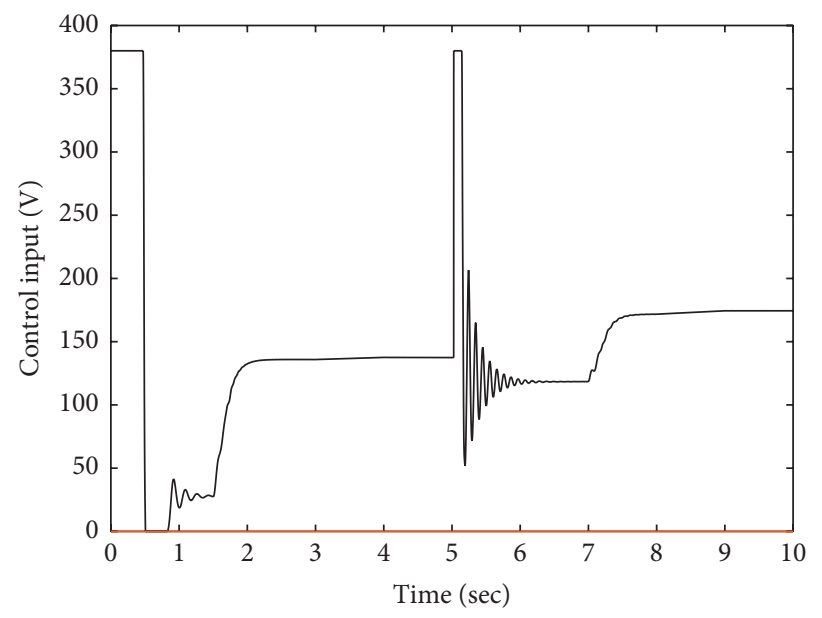

$-u_{d}$

- $u_{q s}$

FIgURE 8: Evolution of optimal control inputs.

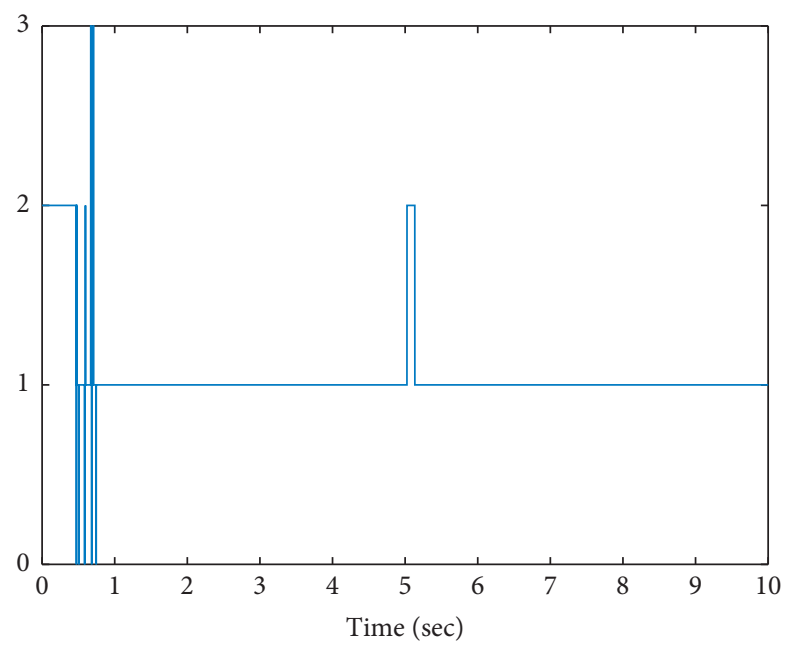

$$
\begin{aligned}
& \text { " } 0 \text { " }-u_{d}=u_{1+} u_{2} \\
& \text { "1" }-u_{d}=u_{2} \\
& \text { "2" }-u_{d}=V_{\max } \\
& \text { “3"- } u_{d}=0
\end{aligned}
$$

Figure 9: The components of control input on $d$-axis.

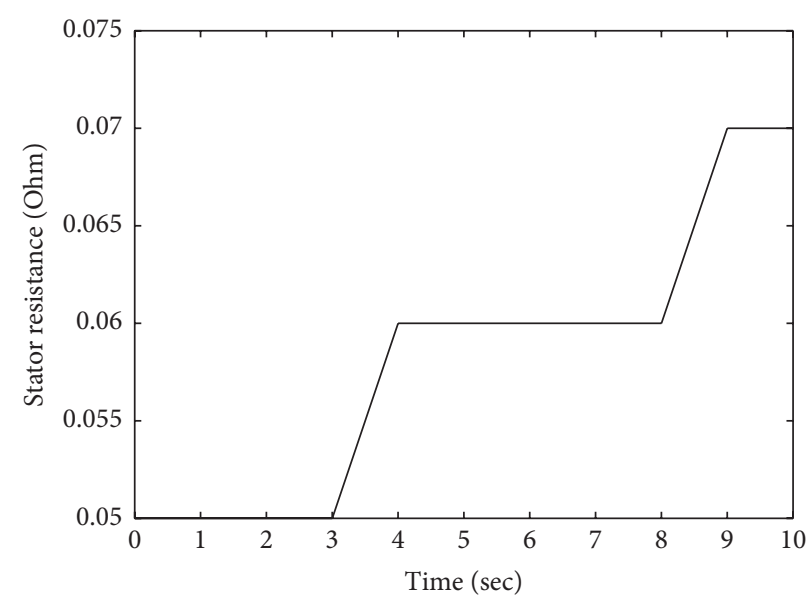

FIgURE 10: Time profile of the stator resistance. 


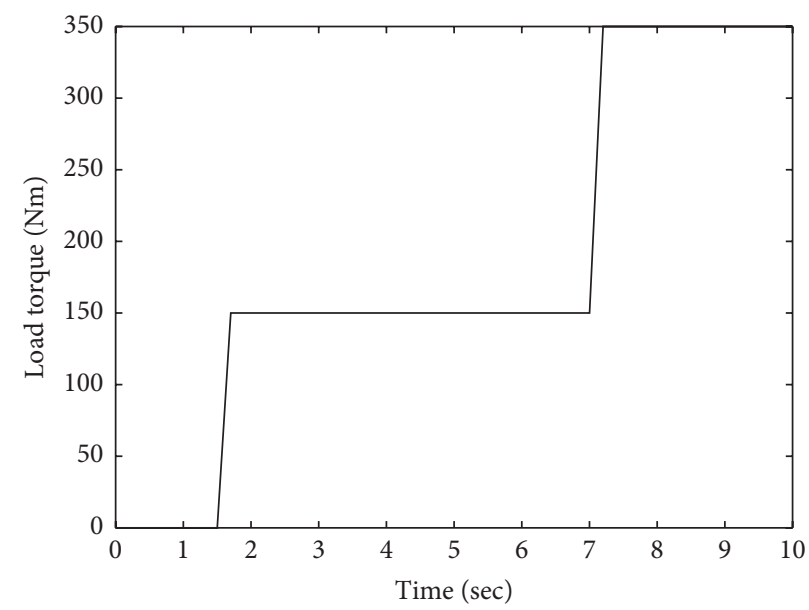

Figure 11: Time-varying profile of the load torque.

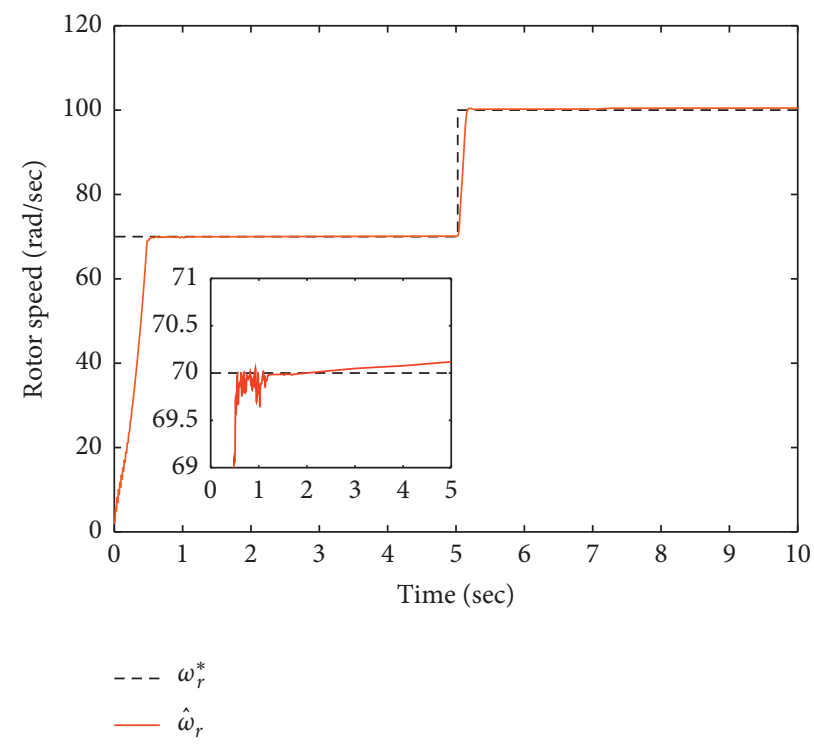

FIGURE 12: Evolution of output-super-twisting control (noisy measurements).

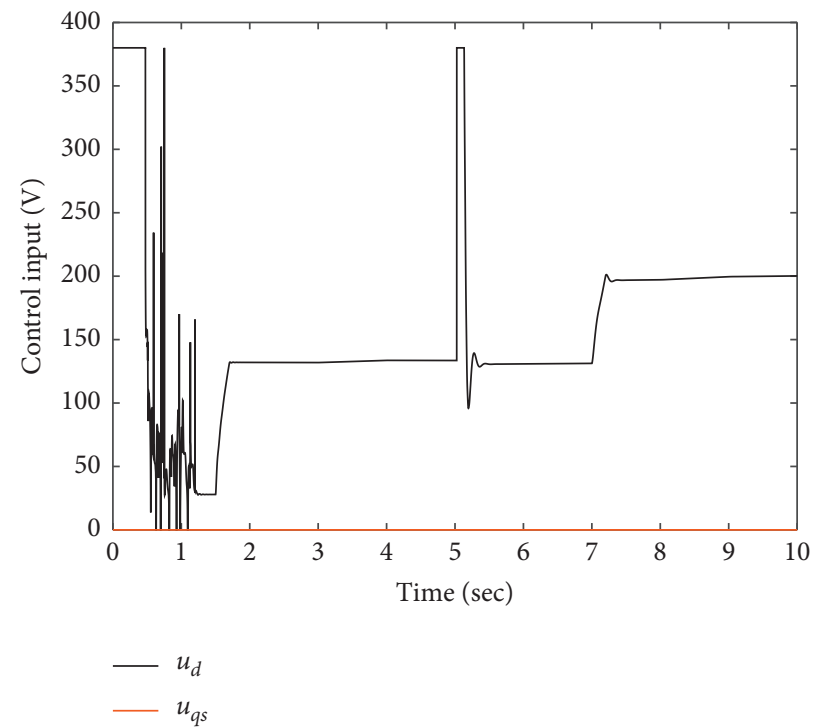

FIGURE 13: Time profiles of control inputs (noisy measurements). 


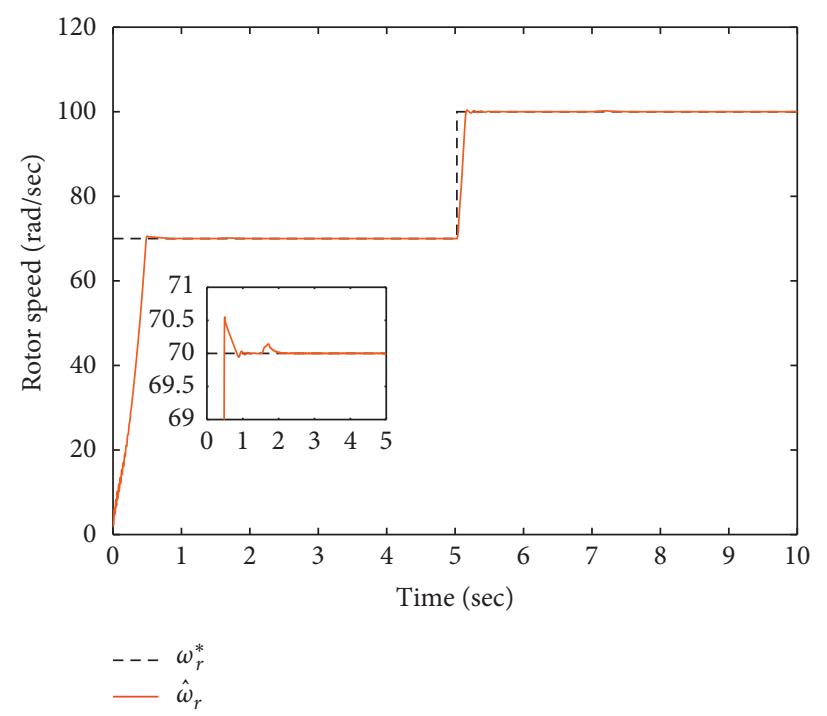

FIGURE 14: Evolution of output-predictive controller (noisy measurements).

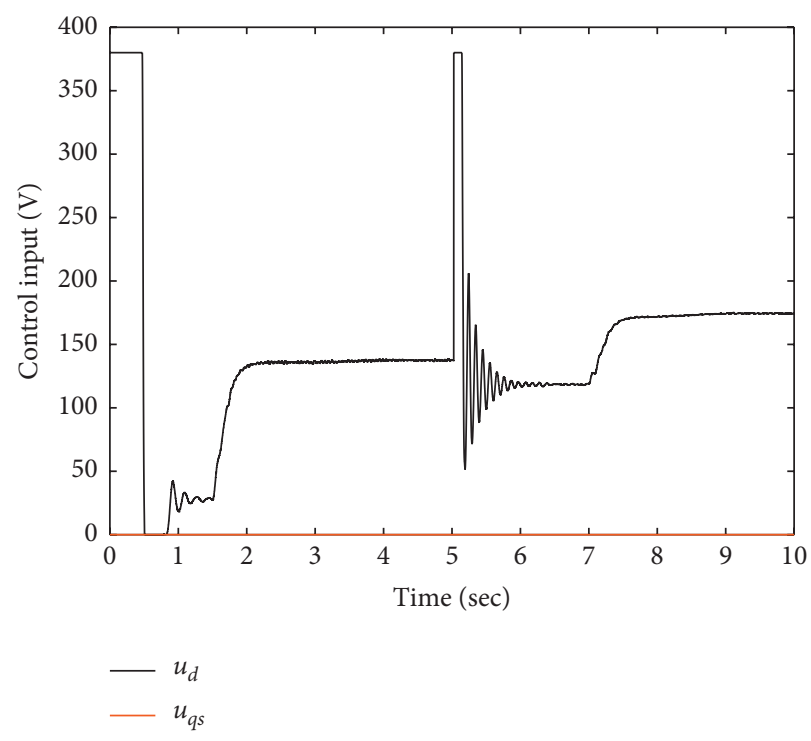

FiguRE 15: Time profile of control input (noisy measurements).

(ii) Mean absolute magnitude of the error, MAE $=(1 / n) \sum_{k=1}^{n}\left|e_{y}\left(k T_{e}\right)\right|$, where $T_{f}$ is the total simulation time, and $n=\left(T_{f} / T_{e}\right)$

The obtained values of the abovementioned metrics are highlighted in Table 1. Both from graphical representations of closed loop behaviour of the system and from the performances indices presented in Table 1, we pointed out that the best results concerning the control objective were obtained by using the predictive control. Also, the proposed super-twisting algorithm provides better results in terms of tracking and robustness problems.

The proposed estimation and control strategies can be applied to many practical situations involving the use of an induction motor, mostly in a sensorless layout. The rotor speed is controlled with partial data provided by the hardware sensors and by using the software sensors to provide the unknown or unmeasurable variables of the process. 


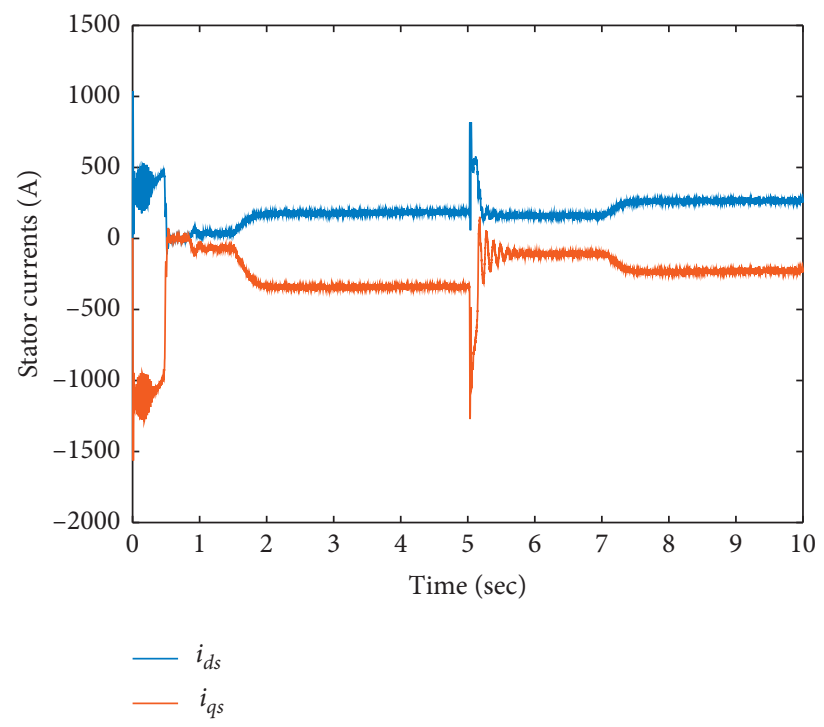

FIgURE 16: Time profiles of stator currents.

TABLE 1: Performance criteria results.

\begin{tabular}{lcccc}
\hline Performance indices & Modified STA & Modified STA (noisy measurements) & Predictive control & Predictive control (noisy measurements) \\
\hline ITAE & 29.19 & 29.27 & 15.14 & 15.29 \\
MAE & 2.34 & 2.35 & 2.16 & 2.17 \\
\hline
\end{tabular}

\section{Conclusions}

The present work approached two realistic control strategies dedicated to sensorless induction motors. Two complex robust controllers were proposed: a modified super-twisting adaptive (STA) technique and a model predictive (MPC) technique. The STA approach used an adaptive gain for the sliding surface to handle the time-varying disturbances acting on the system. The MPC used an objective function that casts the disturbances variable, obtaining in this way an improved robustness of the controller.

Due to the lack of useful measurements, some specific observers were designed in order to successfully implement the robust controllers. More precisely, an innovative linked observer-estimator-was designed and used to reconstruct the rotor fluxes. Also, a parameter estimator and a disturbance observer were developed to cope with parameter uncertainties and load torque estimation.

The overall estimation and control schemes were tested under several practical assumptions concerning the induction motor. The behaviour of the closed loop system for both robust control schemes is satisfactory, taking into account the realistic and harsh simulation scenarios.

The simulation results and the computed performances indices showed that the best control results were obtained in the case of MPC, which provided better results from the robustness point of view.

\section{Data Availability}

The data used to support the findings of this study are available from the corresponding author upon request.

\section{Conflicts of Interest}

The authors declare that they have no conflicts of interest.

\section{Acknowledgments}

This work was supported by the European Regional Development Fund, through the Competitiveness Operational Program (TISIPRO project, ID: P_40_416/105736, 2016-2021).

\section{References}

[1] F. Blaschke, "The principle of field orientation as applied to the new transvector closed loop system for rotating field machines," Siemens Review, vol. 39, no. 5, pp. 217-220, 1972.

[2] P. C. Krause, Analysis of Electric Machinery, McGraw-Hill, New York, NY, USA, 1986.

[3] W. Leonhard, Control of Electrical Drives, Springer-Verlag, Berlin, Germany, 3rd edition, 2001.

[4] J. Chiasson, Modelling and High-Performance Control of Electric Machines, Wiley, Hoboken, NJ, USA, 2005.

[5] R. Marino, P. Tomei, and C. Verrelli, Induction Motor Control Design, Springer-Verlag, London, UK, 2010.

[6] L. Grune and J. Pannek, Nonlinear Model Predictive Control, Springer-Verlag, London, UK, 2011.

[7] B. K. Bose and N. R. Patel, "Quasi-fuzzy estimation of stator resistance of induction motor," IEEE Transactions on Power Electronics, vol. 13, no. 3, pp. 401-409, 1998.

[8] G. Kenné, R. S. Simo, F. Lamnabhi-Lagarrigue, A. Arzandé, and J. C. Vannier, "An online simplified rotor resistance estimator for induction motors," IEEE Transactions on Control Systems Technology, vol. 18, no. 5, pp. 1188-1194, 2010. 
[9] E. Petre, D. Selișteanu, and M. Roman, "Nonlinear robust adaptive control strategies for a lactic fermentation process," Journal of Chemical Technology \& Biotechnology, vol. 93, no. 2, pp. 518-526, 2018.

[10] A. Paladugu and B. H. Chowdhury, "Sensorless control of inverter-fed induction motor drives," Electric Power Systems Research, vol. 77, no. 5-6, pp. 619-629, 2007.

[11] D. Traoré, A. Glumineau, and J. de Leon, "Sensorless induction motor adaptive observer-backstepping controller: experimental robustness tests on low frequencies benchmark," IET Control Theory \& Applications, vol. 4, no. 10, pp. 1989-2002, 2010.

[12] J. Guzinski and H. Abu-Rub, "Speed sensorless induction motor drive with predictive current controller," IEEE Transactions on Industrial Electronics, vol. 60, no. 2, pp. 699-709, 2013.

[13] F. Stîngă and M. Marian, "Estimation and nonlinear predictive control for an induction machine," in Proceedings of the IEEE 6th 2019 International Conference on Control, Decision and Information Technologies, Paris, France, 2019.

[14] G. Bastin and D. Dochain, On-Line Estimation and Adaptive Control of Bioreactors, Elsevier, Amsterdam, Netherlands, 1990.

[15] D. Selişteanu, E. Petre, M. Roman, and D. Şendrescu, "Estimation of kinetic rates in a Baker's yeast fed-batch bioprocess by using nonlinear observers," IET Control Theory \& Applications, vol. 6, no. 2, pp. 243-253, 2012.

[16] K. Ohishi, M. Nakao, K. Ohnishi, and K. Miyachi, "Microprocessor-controlled DC motor for load-insensitive position servo system," IEEE Transactions on Industrial Electronics, vol. IE-32, no. 1, pp. 44-49, 1987.

[17] J. Yang, W.-H. Chen, and S. Li, "Non-linear disturbance observer-based robust control for systems with mismatched disturbances/uncertainties," IET Control Theory \& Applications, vol. 5, no. 18, pp. 2053-2062, 2011.

[18] A. Levant, "Sliding order and sliding accuracy in sliding mode control," International Journal of Control, vol. 58, no. 6, pp. 1247-1263, 1993.

[19] C. E. Zavala, J. A. Moreno, and L. M. Fridman, “Adaptive gains super-twisting algorithm for systems with growing perturbations," in Proceedings of the 18th IFAC World Congress, Milano, Italy, August 2011.

[20] I. Nagesh and C. Edwards, "A multivariable super-twisting sliding mode approach," Automatica, vol. 50, no. 3, pp. 984-988, 2014.

[21] J. A. Moreno and M. A. Osorio, "A Lyapunov approach to second order sliding mode controllers and observers," in Proceedings of the 47th IEEE Conference on Decision and Control, Cancún, Mexico, December 2008.

[22] J. R. Dominguez, L. Garcia, C. Mora, J. Panduro, and S. Ortega, "Super-twisting sliding mode in motion control systems," in Sliding Mode Control, pp. 237-254, In-TechOpen, London, UK, 2011.

[23] O. Morfin, C. Castañeda, A. Valderrabano-Gonzalez, M. Hernandez-Gonzalez, and F. Valenzuela, "A real-time SOSM super-twisting technique for a compound DC Motor velocity controller," Energies, vol. 10, no. 9, p. 1286, 2017.

[24] M. Jouini, S. Dhahri, and A. Sellami, "Design of robust super twisting algorithm based second-order sliding mode controller for nonlinear systems with both matched and unmatched uncertainty," Complexity, vol. 2017, Article ID 1972921, 8 pages, 2017.
[25] Z. Feng and J. Fei, "Design and analysis of adaptive supertwisting sliding mode control for a microgyroscope," PLoS One, vol. 13, no. 1, Article ID e0189457, 2018.

[26] J. A. Rossiter and Y. Ding, "Interpolation methods in model predictive control: an overview," International Journal of Control, vol. 83, no. 2, pp. 297-312, 2010.

[27] N. Chen, M. Wang, T. Alkim, and B. van Arem, "A robust longitudinal control strategy of platoons under model uncertainties and time delays," Journal of Advanced Transportation, vol. 2018, Article ID 9852721, 13 pages, 2018.

[28] H. Shi, L. Ping, L. Wang, C. Su, J. Yu, and J. Cao, "Delayrange-dependent robust constrained model predictive control for industrial processes with uncertainties and unknown disturbances," Complexity, vol. 2019, Article ID 2152014, 15 pages, 2019.

[29] E. F. Camacho and C. Bordons, Model Predictive Control, Springer, Berlin, Germany, 2nd edition, 2007.

[30] T. T. Long and D. Zhengtao, "Reduced-order observer design of multi-output nonlinear systems with application to a circadian model," Transactions of the Institute of Measurement and Control, vol. 35, no. 4, pp. 417-425, 2012.

[31] R. Oliveira, E. C. Ferreira, and S. Feyo de Azevedo, "Stability, dynamics of convergence and tuning of observer-based kinetics estimators," Journal of Process Control, vol. 12, no. 2, pp. 311-323, 2002.

[32] H. M. Kojabadi and L. Chang, "Comparative study of pole placement methods in adaptive flux observers," Control Engineering Practice, vol. 13, no. 6, pp. 749-757, 2005.

[33] F. Stîngă and D. Danciu, "A disturbance observer-based control of drilling vibrations," in Proceedings of the 20th International Carpathian Control Conference (ICCC), Krakow, Poland, 2019.

[34] L. Wang, Model Predictive Control System Design and Implementation Using MATLAB, Springer-Verlag, London, UK, 2009.

[35] D. Q. Mayne, J. B. Rawlings, C. V. Rao, and P. O. M. Scokaert, "Constrained model predictive control: stability and optimality," Automatica, vol. 36, no. 6, pp. 789-814, 2000.

[36] MathWorks, Inc., "Optimization toolbox-user's guide," 2019, https://www.mathworks.com/help/optim/index.html.

[37] T. Chai and R. R. Draxler, "Root mean square error (RMSE) or mean absolute error (MAE)? Arguments against avoiding RMSE in the literature," Geoscientific Model Development, vol. 7, no. 3, pp. 1247-1250, 2014.

[38] W. S. Levine, Control System Fundamentals, CRC Press, Boca Raton, FL, USA, 2011. 